\title{
Statistics of dynamic fragmentation for a necking instability
}

\author{
Jean-Lin Dequiedt \\ CEA, DAM, DIF, 91297 Arpajon, France
}

\begin{abstract}
In the aim of predicting the fragmentation of expanding structures, a Mott type approach is applied to the potential failure points driven from an instability analysis. Substantially, fragmentation is preceded by the localization of plastic deformation in multiple necks forming potential sites for failure. This pattern is predicted by a combination of periodic instable eigenmodes of the structure, the necks being the maxima with varying spacing and amplitudes of the instable perurbation. Obscuration conditions, linked to the propagation of unloading waves, are next written for this set of potential failure points and yield a distribution of fragments. The approach is developed for an expanding ring experiment justifying the scatter in fragment sizes. It shows incidentally that the unloading waves must propagate at a celerity much lower than the elastic wave speed.
\end{abstract}

\section{Introduction}

The fragmentation of structures under dynamic expansion is governed by the competition between the activation of failure points when deformation increases and the inhibition of failure in areas obscured by the unloading waves emerging from these points. The phenomenon has been modeled with various assumptions since the early work of Mott [1], usually postulating random positions in the structure for the potential failure sites [2]. It yielded statistic distributions for the fragment sizes at the end of the fragmentation process. However, for ductile materials, fragmentation is initiated by the localization of plastic deformation and these sites are clearly related with the necking pattern emerging in this first phase. The onset of localization is ruled by the existence of instable eigenmodes of the structure and it is treated in a linear stability analysis framework. The aim of the present work is to apply the approach of Mott to a discrete set of potential failure points identified as the maxima of an instable perturbation (forming necks in thickness of the structure): a combination of instable modes is retained in order to get maxima of various amplitudes and spacing as shown in [3]. This justifies the scatter in failure times which is at the origin of the obscuration process. The one dimensional case of an expanding ring, assimilated to a stretching rod, is developed and, as an illustration, the predictions are compared to the sizes of fragments recovered after an explosive expansion experiment.

\section{Potential failure points generated by a plastic necking instability}

The linear stability analysis of a stretching cylindrical rod representing an expanding ring (Fig. 1) is conducted in a one-dimensional framework as in Zhou et al. [4] in order to identify the instable eigenmodes of the structure and next the positions and failure times of the potential failure points associated with a multimode perturbation. The rod

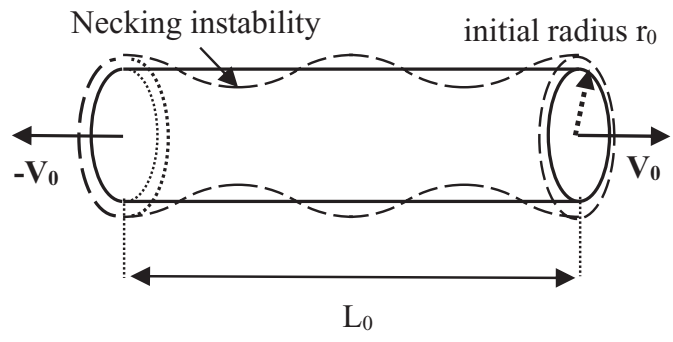

Figure 1. Stretching rod and necking instability.

is submitted to a constant tensile velocity at the extremities and the initial strain rate is $\dot{\varepsilon}_{0}=V_{0} / L_{0}$. The material is supposed to be incompressible with mass density $\rho$ and strain hardening. Elasticity and thermal effects are neglected in the localization phase. All mechanical variables are functions of the Lagrangian coordinate $X$ along the rod and of time $t$.

The constitutive relations satisfied by the homogeneous and perturbed response of the rod are obtained in the following way. The yield stress is supposed to be a function of the logarithmic strain $\left(\sigma_{Y}(\varepsilon)\right)$ and the axial stress is derived by taking into account the triaxiality of stress in necked regions with the formula of Bridgman [5]:

$$
\sigma=\left(1+\frac{1}{\theta}\right) \ln (1+\theta) \sigma_{Y}
$$

in which $\theta$ measures the local curvature of the rod external profile. It expresses as a function of the strain derivatives and the initial radius $r_{0}$ of the rod cross section:

$$
\theta=\frac{r_{0}^{2}}{8}\left(-2\left(\frac{d^{2} \varepsilon}{d X^{2}}\right)+\left(\frac{d \varepsilon}{d X}\right)^{2}\right) \exp (-\varepsilon)
$$

The strain and material velocity satisfy:

$$
\frac{d v}{d X}=\exp (\varepsilon) \frac{d \varepsilon}{d t}
$$

This is an Open Access article distributed under the terms of the Creative Commons Attribution License 4.0, which permits unrestricted use, distribution, and reproduction in any medium, provided the original work is properly cited. 
and the momentum balance writes:

$$
\frac{d}{d X}(\sigma \exp (-\varepsilon))=\rho \frac{d v}{d t} .
$$

Instable perturbations $(\delta \varepsilon, \delta v)$ of the uniform stretching of the rod are searched in the form of periodic eigenmodes growing exponentially with time:

$$
\begin{aligned}
& \delta \varepsilon(X, t)=\delta \hat{\varepsilon} \exp (i k X+\eta t) \\
& \delta v(X, t)=\delta \hat{v} \exp (i k X+\eta t) .
\end{aligned}
$$

Equations (1) to (4) yields to the following relation between the growth rates and wavenumbers of perturbations at any time $t_{0}$ :

$$
\begin{aligned}
& \eta^{2}-\frac{1}{\rho} k^{2} \exp \left(-2 \varepsilon\left(t_{0}\right)\right)\left(\sigma_{Y}\left(t_{0}\right)-\frac{d \sigma_{Y}}{d \varepsilon}\left(t_{0}\right)\right) \\
& +\frac{r_{0}^{2}}{8 \rho} k^{4} \exp \left(-3 \varepsilon\left(t_{0}\right)\right) \sigma_{Y}\left(t_{0}\right)=0 .
\end{aligned}
$$

The curve $\eta(k)$ evolves with time $t_{0}$ but all curves are similar with a maximum $\eta_{\max }\left(t_{0}\right)$ for a wavenumber $k_{\max }\left(t_{0}\right)$ (or wavelength $\lambda_{\max }\left(t_{0}\right)$ ):

$$
\frac{\eta(k)}{\eta_{\max }}=\sqrt{2\left(\frac{k}{k_{\max }}\right)^{2}-\left(\frac{k}{k_{\max }}\right)^{4}}
$$

with:

$$
\begin{aligned}
& k_{\max }\left(t_{0}\right)=\frac{2}{r_{0}} \exp \left(\frac{\varepsilon\left(t_{0}\right)}{2}\right) \sqrt{1-\frac{1}{\sigma_{Y}\left(t_{0}\right)} \frac{d \sigma_{Y}}{d \varepsilon}\left(t_{0}\right)} \\
& \eta_{\max }\left(t_{0}\right)= \frac{\sqrt{2}}{r_{0}} \sqrt{\frac{\sigma_{Y}\left(t_{0}\right)}{\rho}} \exp \left(-\frac{\varepsilon\left(t_{0}\right)}{2}\right) \\
& \times\left(1-\frac{1}{\sigma_{Y}\left(t_{0}\right)} \frac{d \sigma_{Y}}{d \varepsilon}\left(t_{0}\right)\right) .
\end{aligned}
$$

The instable perturbations belong to the domain of positive $\eta(k)$, namely of wavenumbers $k<\sqrt{2} k_{\max }$. Let us assume that the whole localization process is governed by the spectrum of instable modes identified at time $t_{0}$ and that these modes develop much faster than the homogeneous solution, in such a way that the time evolution of the growth rates can be ignored. The fastest growing mode $\left(\eta_{\max }\right)$ is usually considered as dominating the localization process and $\lambda_{\max }$ gives an estimation of the average neck spacing.

The circumference of the ring is supposed to be $N_{L} \lambda_{\max }$ and the instable perturbation to be initiated by a "white noise", i.e. a combination of all instable modes with wavenumbers $k_{j}=\left(j / N_{L}\right) k_{\max }$, equal amplitudes and arbitrary phases $\varphi_{j}$. The perturbation is thus a multimode one with amplitudes growing in accordance with the respective growth rates of the modes (represented on Fig. 2). The time evolution of this perturbation then writes:

$$
\begin{aligned}
& \delta \varepsilon(X, t)=\frac{\delta \varepsilon_{0}}{\sqrt{E\left(\sqrt{2} N_{L}\right)}} \\
& \times \sum_{j=1}^{E\left(\sqrt{2} N_{L}\right)} \cos \left(k_{j} X+\varphi_{j}\right)^{*} \exp \left(\eta\left(k_{j}\right) t\right) .
\end{aligned}
$$

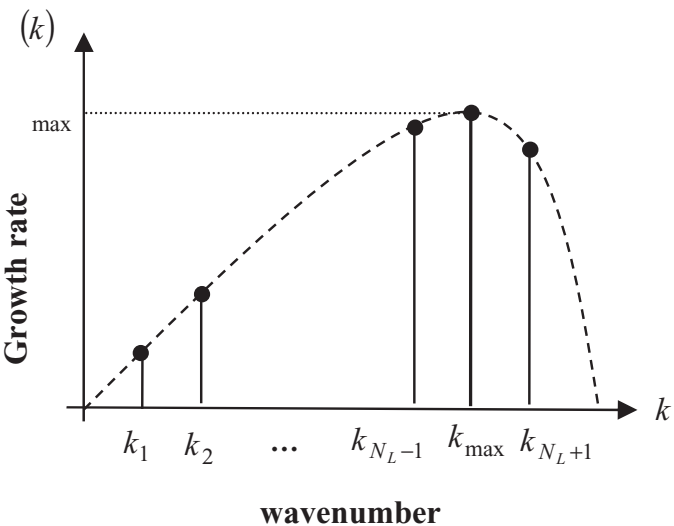

Figure 2. Spectrum of the instable perturbation and growth rates.

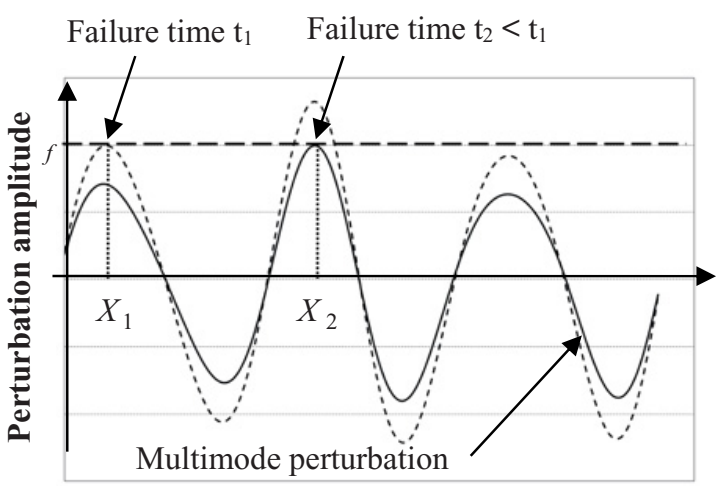

Position

Figure 3. Determination of failure points for a multimode perturbation.

The perturbation has maxima with amplitudes and spacing varying with time.

The failure of the rod is ruled by a critical value $\delta \varepsilon_{f}$ for the perturbation in strain. One potential failure point is identified each time that a maximum of the perturbation reaches $\delta \varepsilon_{f}$. The failure points $X_{l}$ and failure times $t_{l}$ thus satisfy (as schematized on Fig. 3):

$$
\begin{aligned}
& \delta \varepsilon\left(X_{l}, t_{l}\right)=\delta \varepsilon_{f} \quad \text { and } \quad \frac{d \delta \varepsilon}{d X}\left(X_{l}, t_{l}\right)=0 \\
& \left(\text { such that: } \frac{d^{2} \delta \varepsilon}{d X^{2}}\left(X_{l}, t_{l}\right)<0\right) .
\end{aligned}
$$

These points depend, among others, on the ratio between the failure strain $\delta \varepsilon_{f}$ and the initial amplitude of the perturbation $\delta \varepsilon_{0}$.

\section{Fragmentation of a discrete set of failure points}

Let us consider a discrete set of potential failure points with different failure times. When one of these points breaks, two unloading waves are emitted on both sides. They are supposed to propagate at a constant celerity $c$. They cover a so-called "obscuration cone" in the $(X, t)$ plane in which failure is inhibited [6]. It can be easily seen that the obscuration cone of a point contains the 


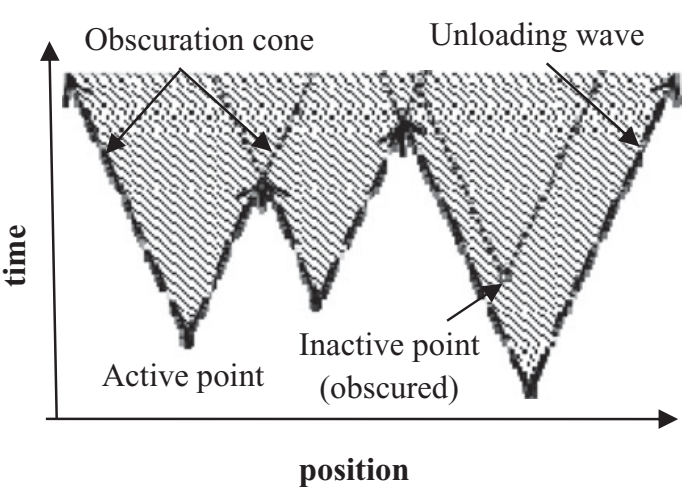

Figure 4. Area obscured by a family of failure points.

obscuration cones of all the points it obscures. Therefore, the area obscured by a set of points is the union of all obscuration cones no matter whether the points are active or not (Fig. 4).

One point $l$ actually breaks if $\left(X_{l}, t_{l}\right)$ is outside the area obscured by all the preceding and following points, namely if:

$$
\begin{aligned}
& t_{l-p}+\frac{1}{c}\left|X_{l}-X_{l-p}\right|>t_{l} \quad \text { and } \\
& t_{l+p}+\frac{1}{c}\left|X_{l}-X_{l+p}\right|>t_{l}
\end{aligned}
$$$$
\text { for any } p>0 \text {. }
$$

\section{Fragment size distribution for an expanding copper ring}

An expanding copper ring experiment designed by CEA/Valduc is considered [7]. The potential failure points are identified from the analysis of the instable modes of the ring. The obscuration conditions are written in order to estimate which of these points actually break and comparison is made with the recovered fragments.

More precisely, the experimental set-up is given in Fig. 5. The ring deforms under free expansion after separating from the transmitter and multiple necks progressively appear. Some of these necks concentrate the deformation up to failure. The fragments were recovered after the test and allowed to reconstitute the entire ring with 7 failing points and 7 arrested necks (which could be interpreted as obscured failure points). The final elongation was about $46 \%$ giving an estimate of the ring deformation at the onset of necking.

The experiment is simulated and the evolution of the instable eigenmodes with time is investigated (namely of $k_{\max }\left(t_{0}\right)$ and $\eta_{\max }\left(t_{0}\right)$ given by (8) and (9)).

The localization process is supposed to be governed by the modes growing around time $t_{0}$ such that circumference of the ring is 14 times the dominant wavelength $\lambda_{\max }\left(t_{0}\right)$ (which would give an average of 14 necks in accordance with experiment). Then, the failure points associated with the multimode perturbation given by relation (10) and a random set of phases are displayed (a ratio $\delta \varepsilon_{f} / \delta \varepsilon_{0}=1000$ is assumed). Their positions and failure times are reported on Fig. 6.

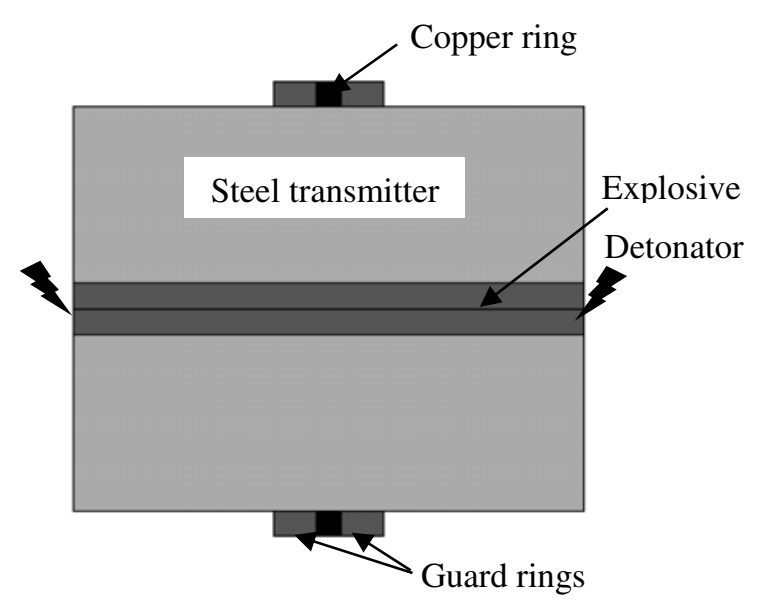

Figure 5. Expanding ring set-up.

(a)

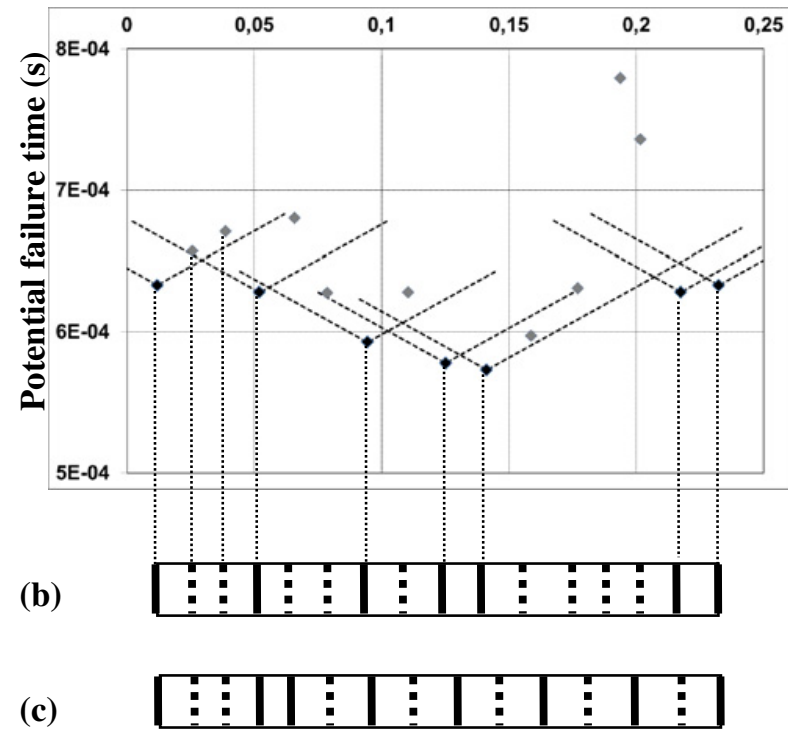

Figure 6. (a) Potential failure points and unloading waves (dashed lines) for the copper ring (active points in black and obscured points in gray). Distribution of broken and arrested (dotted) necks in (b) the prediction and (c) the experiment.

The obscuration process is next investigated by considering different values for the unloading wave celerity.

The most intuitive is to consider that they are elastic waves as in [8]; then the celerity in uniaxial stress is given by $c=\sqrt{E / \rho} \approx 3700 \mathrm{~m} / \mathrm{s}$ where $E$ is the Young modulus. However, this leads to unloading waves propagating very fast in such a way that the first breaking neck would obscure all the others, which does not match with experimental results. Thus, the celerity is progressively reduced down to $c=1000 \mathrm{~m} / \mathrm{s}$ when about a half of the necks are inhibited.

In this case, the release waves are plotted and the active and obscured points are identified on Fig. 6. Below, the predicted distribution of broken and arrested necks is drawn and compared with the experimental one. 
It comes that both experiment and prediction exhibit heterogeneity of fragment sizes but less pronounced in the experiment. More generally, the influence of many parameters such as the ratio $\delta \varepsilon_{f} / \delta \varepsilon_{0}$ or the spectrum of the initial perturbation should be addressed more precisely before rigorous comparison with experiment really makes sense. Incidentally, the sensitivity to the set of phases, not illustrated here, may explain a part of the differences.

\section{Conclusion}

The prediction of the fragment size distribution resulting from the fragmentation of a ductile expanding structure may be addressed by coupling an instability analysis to predict the onset of localization and a fragmentation approach to investigate the obscuration process governing the inhibition of some weak points.

Some elements are given by showing that a multimode linear analysis yields to the formation of necks of varying amplitudes which may break at different times. It gives rise to the formation of unloading waves obscuring the necks with the latest failure times.

Comparison with experiment, although depending on many assumptions, seems to show, however, that the unloading waves cannot propagate as fast as the elastic wave speed, which is consistent with the progressive unloading observed in the simulation of necking [9].
At last, the stochastic character of failure, usually assumed in fragmentation analyses, has been disregarded here. It would introduce an additive scatter of failure times and favor obscuration.

\section{References}

[1] N.F. Mott, In: Proceedings of the Royal Society. A, 300-308, 1947

[2] D.E. Grady, J. Geophys. Res., 86, B2, 1047-1054, 1981

[3] S. El Maï, S. Mercier, J. Petit, A. Molinari, Int. J. Sol. Struct., 51, 3491-3507, 2014

[4] F. Zhou, J.F. Molinari, K.T. Ramesh, Int. J. Imp. Eng., 33, 880-891, 2006

[5] P.W. Bridgman, Studies in large plastic flow and fracture, with special emphasis on the effect of hydrostatic pressure (McGrawHill Book Company Inc., New York, 1952)

[6] C. Denoual, F. Hild, Eur. J. Mech. A/Solids, 21, 105-120, 2002

[7] F. Llorca, A. Juanicotena, In: J. Phys IV France 7, C3, 235-240, 1997

[8] E.H. Lee, Energetics in Metallurgical Phenomena III (Ed. W.M. Mueller, M. Shaw, Gordon and Breach, New York, 1967)

[9] F. Dalle, In: J. Phys IV France, 134, 429-434, 2006 